\title{
PRECISE ESTIMATION ON THE ORDER OF LOCAL TESTABILITY OF DETERMINISTIC FINITE AUTOMATON
}

\author{
A.N. Trahtman \\ Bar-Ilan University, Department of Mathematics and Computer Science, 52900 \\ Ramat Gan,Israel, e-mail: trakht@macs.biu.ac.il \\ Lectures Notes of Computer Sciences, 1436(1998), 198-212 \\ AUTOMATA IMPLEMENATION
}

\begin{abstract}
A locally testable language $L$ is a language with the property that for some nonnegative integer $k$, called the order or the level of local testability, whether or not a word $u$ in the language $L$ depends on (1) the prefix and suffix of the word $u$ of length $k-1$ and (2) the set of intermediate substrings of length $k$ of the word $u$. For given $k$ the language is called $k$-testable.

We give necessary and sufficient conditions for the language of an automaton to be $k$-testable in the terms of the length of paths of a related graph. Some estimations of the upper and of the lower bound of order of testability follow from these results.

We improve the upper bound on the order of testability of locally testable deterministic finite automaton with $n$ states to $\frac{n^{2}-n}{2}+1$. This bound is the best possible.

We give an answer on the following conjecture of Kim, McNaughton and McCLoskey for deterministic finite locally testable automaton with $n$ states: "Is the order of local testability no greater than $\Omega\left(n^{1.5}\right)$ when the alphabet size is two?"

Our answer is negative. In the case of size two the situation is the same as in general case: the order of local testability is $\Omega\left(n^{2}\right)$.
\end{abstract}

Key words: finite automaton, language, semigroup, identity, locally testable, order of local testability, algorithm

\section{Introduction}

The concept of local testability was first introduced by McNaughton and Papert [9] and since then has been extensively investigated from different points of view (see [1], [3], [4] - [6], [8], [11], [12], [14], [16], [17]). This concept is connected with languages, finite automata and semigroups. In [10], local testability is discussed in terms of "diameter-limited perceptrons". Locally testable languages 
are a generalization of the definite and reverse-definite languages, which can be found, for example, in [2] and [13].

In [5] necessary and sufficient conditions for an automaton to be locally testable were found. In [6] the NP-hardness of finding of the order of local testability was proved. The necessary and sufficient conditions of $k$-testability in the terms of 5-tuple graph were found in [6]. An estimation for the order of local testability for an arbitrary deterministic finite automaton was found first in [5] and then improved in [6]. The upper bound from [6] is $2 n^{2}+1$, where $n$ is the number of states of the automaton.

For the state transition graph $\Gamma$ of an automaton we consider some subgraphs of the direct product $\Gamma \times \Gamma$. We introduce in this paper sufficient and necessary conditions for the automaton and transition semigroup of the automaton to be $k$-testable in terms of the length of some paths without loops on these graphs. This gives us some upper and some lower bounds on the order of local testability.

In the case that the state transition graph is strongly connected the sufficient conditions are necessary as well and algorithm of finding of the level of local testability is polynomial and not NP-hard as in the general case [6].

As corollary we receive the precise upper bound on the order of local testability for deterministic finite locally testable reduced automaton with $n$ states. It is equal to $\left(n^{2}-n\right) / 2+1$. This result improves the estimations from [5], [6] and finishes investigations in this direction.

In [4] and [6] one can find conjecture that in the case of the alphabet two the upper bound on the order of local testability for the deterministic finite locally testable reduced automaton with $n$ states is not greater than $\Omega\left(n^{1.5}\right)$.

We consider in this paper an example of sequence of deterministic finite automata with $n$ states whose alphabet size is two. It will be proved that the considered automata are locally testable and their order of local testability is $\Omega\left(n^{2}\right)$. So the problem from [4], [6] is solved negatively.

Our example is one between examples of locally testable automata whose order of testability is greater than the number of its states. First such astonishing example of an automaton with 28 states had appeared in [4] - [6]. (Note that the order of testability of the considered automaton found in these papers is not correct. It is more greater than 126 [4] or 127 [6]. The conjuncture of the authors that the automaton has the maximal order of testability for automata with 28 states and alphabet size two is not correct too. There exist a deterministic finite 142-testable automaton with 28 states and alphabet size two).

The description of the identities of $k$-testable semigroup from [14] is used here. The concept of the graph is inspired by the works [4], [5] of Kim, McNaughton and McCLoskey. The purely algebraic approach proved to be fruitful (see [11], [14], [16]) and in this paper we use this technique too. The results of the work are announced in [15]. 


\section{Notation and definition}

Let $\Sigma$ be an alphabet and let $\Sigma^{+}$denote the free semigroup on $\Sigma$. If $w \in \Sigma^{+}$, let $|w|$ denote the length of $w$. Let $k$ be a positive integer. Let $i_{k}(w)\left[t_{k}(w)\right]$ denote the prefix [suffix] of $w$ of length $k$ or $w$ if $|w|<k$. Let $F_{k}(w)$ denote the set of factors of $w$ of length $k$. A language $L$ [a semigroup $S$ ] is called $k$-testable if there is an alphabet $\Sigma$ [and a surjective morphism $\phi: \Sigma^{+} \rightarrow S$ ] such that for all $u$, $v \in \Sigma^{+}$, if $i_{k-1}(u)=i_{k-1}(v), t_{k-1}(u)=t_{k-1}(v)$ and $F_{k}(u)=F_{k}(v)$, then either both $u$ and $v$ are in $L$ or neither is in $L[u \phi=v \phi]$.

This definition follows [1], [4]. In [9] the definition differs by considering prefixes and suffixes of length $k$.

An automaton is $k$-testable if the automaton accepts a $k$-testable language [the syntactic semigroup of the automaton is $k$-testable].

A language $L$ [a semigroup $S$, an automaton $\mathbf{A}$ ] is locally testable if it is $k$-testable for some $k$.

For local testability the two definitions mentioned above are equivalent [4] .

It is known that the set of $k$-testable semigroups forms a variety of semigroups ([7], [16]). Let $T_{k}$ be the variety of $k$-testable semigroups.

$|S|$ - the number of elements of the set $S$.

$|d|$ - the length of the word $d$ in some alphabet.

$S^{m}$ - the ideal of the semigroup $S$ containing products of elements of $S$ of length $m$ and greater.

We say that the element $a$ from a semigroup $S$ divides the element $b$ from $S$ if $b=d a c$ for some $c, d \in S \cup \emptyset$.

According to the result from [14] $T_{n}$ has the following basis of identities:

$$
\alpha_{r}:\left(x_{1} \ldots x_{r}\right)^{m+1} x_{1} \ldots x_{p}=\left(x_{1} \ldots x_{r}\right)^{m+2} x_{1} \ldots x_{p}
$$

where $r \in\{1, \ldots n\}, p=n-1(\bmod r), m=(n-p-1) / r, n=m r+p+1$,

$$
\beta: x_{1} \ldots x_{n-1} y x_{1} \ldots x_{n-1} z x_{1} \ldots x_{n-1}=x_{1} \ldots x_{n-1} z x_{1} \ldots x_{n-1} y x_{1} \ldots x_{n-1}
$$

For instance, $\alpha_{1}: x^{n}=x^{n+1}$. A locally testable semigroup $S$ has only trivial subgroups [1] and so a locally testable semigroup $S$ with $n$ elements satisfies identity $\alpha_{1}$.

A maximal strongly connected component of the graph will be called $S C C$ $[4]$

Let $\Gamma$ be the state transition graph of a finite automaton with edges labeled by elements of $\Sigma$.

The state transition graph $\Gamma$ of a finite automaton is called complete if for every node $\mathbf{p} \in \Gamma$ and every $\sigma \in \Sigma$ we have $\mathbf{p} \sigma \in \Gamma$. Any state transition graph $\Gamma$ of a finite automaton may be transformed in complete graph by adding sink state.

The element $e \in \Sigma^{+}(\in S)$ will be called right unit of the node $\mathbf{p} \in \Gamma$ if $\mathbf{p} e=\mathbf{p}$.

We shall write $\mathbf{p} \succeq \mathbf{q}$ if the node $\mathbf{q}$ is reachable from the node $\mathbf{p}$ and $\mathbf{p} \succ \mathbf{q}$ if $\mathbf{p} \succeq \mathbf{q}$ and the nodes $\mathbf{p}, \mathbf{q}$ are distinct. 
In the case $\mathbf{p} \succeq \mathbf{q}$ and $\mathbf{q} \succeq \mathbf{p}$ we write $\mathbf{p} \sim \mathbf{q}(\mathbf{p}$ and $\mathbf{q}$ belong to one $S C C)$.

We construct now a edge-labeled directed graph $\Gamma \Gamma$ on the nodes $(\mathbf{p}, \mathbf{q})$ where $\mathbf{p}, \mathbf{q} \in \Gamma$ and $\mathbf{p} \succ \mathbf{q}$. We say $(\mathbf{p}, \mathbf{q}) \rightarrow(\mathbf{r}, \mathbf{t})$ iff for some $\sigma \in \Sigma$ we have $\mathbf{p} \sigma=\mathbf{r}$ and $\mathbf{q} \sigma=\mathbf{t}$. The corresponding edge in $\Gamma \Gamma$ will be labeled by $\sigma$. The graph $\Gamma \Gamma$ will be called the 2-tuple graph of the automaton.

The path $\Phi$ on the 2-tuple graph $\Gamma \Gamma$ will be called $S C C$-restricted if all components of its nodes belong to one $S C C$ of $\Gamma$.

Consider a path $\Phi:\left(\mathbf{p}_{1}, \mathbf{q}_{1}\right), \ldots\left(\mathbf{p}_{k}, \mathbf{q}_{k}\right)$ on the 2-tuple graph for which there exist $\sigma \in \Sigma$ such that $\mathbf{p}_{k} \sigma \nsucc \mathbf{q}_{k} \sigma$ and $\mathbf{q}_{k} \sigma \succ \mathbf{q}_{1}$ on the graph $\Gamma$. Note that all $\mathbf{q}_{\mathbf{i}}$ belong to one $S C C$ of $\Gamma$. The path $\Phi$ will be called a $S C C$-semirestricted path.

Consider a path $\Phi$ on the graph $\Gamma \Gamma$ with the nodes $\left(\mathbf{a}_{1}, \mathbf{b}_{1}\right),\left(\mathbf{a}_{2}, \mathbf{b}_{2}\right), \ldots$ $\left(\mathbf{a}_{s}, \mathbf{b}_{s}\right)$ such that there exist a natural number $r$ such that $\mathbf{a}_{i+r}=\mathbf{b}_{i}$ for all possible natural $i$ and for each $j$ there exist such $\sigma \in \Sigma$ that for all $i \geq 0$ we have $\left(\mathbf{a}_{j+r i}, \mathbf{b}_{j+r i}\right) \sigma=\left(\mathbf{a}_{j+r i+1}, \mathbf{b}_{j+r i+1}\right)$. The path $\Phi$ will be called $r$-periodic path.

A path without loops is called simple. A path without common nodes with any $S C C$ will be called strongly simple.

The length of a path is the number of edges on the path.

\section{The graph of the automaton}

We present two key lemmas of Kim, McNaughton and McCLoskey in the following convenient form:

Lemma 31 ([4]) Let the nodes $\mathbf{p}, \mathbf{q}$ belong to one SCC of the state transition graph of a locally testable deterministic finite automaton.

Then the node $(\mathbf{p}, \mathbf{q})$ does not belong to some $S C C$ of the 2-tuple graph $\Gamma \Gamma$ of the automaton.

Lemma 32 ( [5], Lemma 4) Let the node ( $\mathbf{p}, \mathbf{q}$ ) belong to some SCC of the 2-tuple graph $\Gamma \Gamma$ of a locally testable deterministic finite automaton and let $s$ be an arbitrary element of the transition semigroup of the automaton.

Then $\mathbf{p} s \succeq \mathbf{q}$ is valid iff $\mathbf{q} s \succeq \mathbf{q}$ on the state transition graph of the automaton.

Both these lemmas give us necessary and sufficient conditions for a deterministic finite automaton to be locally testable [4], [5].

Lemma 33 Let $S$ be transition semigroup of a locally testable reduced deterministic finite automaton and let $\Gamma \Gamma$ be its 2-tuple graph. Suppose for some elements $a_{1}, \ldots a_{r} \in S$ for some nonnegative $m$ and $p<r$ we have $\left(a_{1} \ldots a_{r}\right)^{m+1} a_{1} \ldots a_{p} \neq$ $\left(a_{1} \ldots a_{r}\right)^{m+2} a_{1} \ldots a_{p}$.

Then on the graph $\Gamma \Gamma$ there exist a simple path of the length $m r+p$.

If on the graph $\Gamma \Gamma$ there is no simple path of length $k-1$ then the identities $\alpha_{r}$ (1) of $k$-testability are valid on $S$. 
Proof. It follows from the given inequality that for some node $\mathbf{q}$ from the state transition graph $\Gamma$ we have $\mathbf{q}\left(a_{1} \ldots a_{r}\right)^{m+1} a_{1} \ldots a_{p} \neq \mathbf{q}\left(a_{1} \ldots a_{r}\right)^{m+2} a_{1} \ldots a_{p}$. At least one of two parts of the inequality is a node of $\Gamma$. It implies that $\mathbf{q}\left(a_{1} \ldots a_{r}\right)^{m+1} a_{1} \ldots a_{p}$ is a node of $\Gamma$. Denote the left subword of the word $\left(a_{1} \ldots a_{r}\right)^{n}$ of length $i$ by $b_{i}$. On the graph $\Gamma \Gamma$ there exist a path $\phi$ from the node $\left(\mathbf{q}, \mathbf{q} b_{r}\right)$ to the node $\left(\mathbf{q} b_{i}, \mathbf{q} b_{i+r}\right)$ and its minimum length is $m r+p$. Our aim is now to find on this path a simple subpath of the necessary length.

So suppose that $\phi$ is not simple and there exist a loop on the path $\phi$. Let the nodes on the places $k$ and $k+j$ coincide for the first such loop from the left. So $\mathbf{q} b_{k}=\mathbf{q} b_{k+j}$ and $\mathbf{q} b_{k+r}=\mathbf{q} b_{k+r+j}$. Then the two nodes $\mathbf{q} b_{k}$ and $\mathbf{q} b_{k+r}$ from $\Gamma$ have the same right unit. In view of lemma 3.1 the nodes $\mathbf{q} b_{k}$ and $\mathbf{q} b_{k+r}$ belong to different $S C C$. From lemma 32 it follows that all nodes $\mathbf{q} b_{l}$ for $l \geq k+r$ on the considered path $\phi$ belong to the same $S C C$ of $\Gamma$. If the node $\mathbf{q} b_{m r+r+p}$ exists, then the node $\mathbf{q} b_{m r+2 r+p}$ exist as well. After the node $\left(\mathbf{q} b_{k+r}, \mathbf{q} b_{k+2 r}\right)$ on $\phi$ there are no loops (Lemma 3.1). Hence, $j<r$. There are no loops on the path before node $\left(\mathbf{q} b_{k}, \mathbf{q} b_{k+r}\right)$ by the choice of $k$. We can exclude all possible loops between these two nodes and obtain a subpath without loops.

From the existence of node $\mathbf{q} b_{m r+2 r+p} \in \Gamma$ it follows that the length of the path $\phi$ is $(m+1) r+p$ and the length of this simple subpath will be at least $m r+p+1$.

In the case there are no loops on $\phi$, the length of $\phi$ will be at least $m r+p$. This follows from existence of the node $\mathbf{q} b_{m r+r+p}$.

The first part of the statement of lemma is proved.

Suppose now that on $\Gamma \Gamma$ there are no simple paths of the length $k-1$. Then for $k-1<m r+p$ and for any $\mathbf{q} \in \Gamma$ we have $\mathbf{q}\left(a_{1} \ldots a_{r}\right)^{m+1} a_{1} \ldots a_{p}=$ $\mathbf{q}\left(a_{1} \ldots a_{r}\right)^{m+2} a_{1} \ldots a_{p}$. The second statement of the lemma follows now from the first and from the description of the identities (1) of $k$-testability.

Lemma 34 If on the 2-tuple graph $\Gamma \Gamma$ of a deterministic finite automaton there exist an $r$-periodic path of length $k+r-1$ then the automaton is not $k$-testable.

$k+1$ is a lower bound on the order of local testability of the automaton

Proof. Suppose that the automaton is locally testable. Let $(\mathbf{s}, \mathbf{q})$ be the first node on the considered $r$-periodic path and elements $a_{1}, \ldots a_{r}$ from $\Sigma$ denote the first $r$ edges of the path. So $\mathbf{s} a_{1} \ldots a_{r}=\mathbf{q}$ and the last node on the path is $\left(\mathbf{s}\left(a_{1} \ldots a_{r}\right)^{m+1} a_{1} \ldots a_{p}, \mathbf{s}\left(a_{1} \ldots a_{r}\right)^{m+2} a_{1} \ldots a_{p}\right)$ where $m r+p=k-1, p<r$, $m \geq 0$. Number of the edges on the path is $(m+1) r+p=k+r-1$. The components of the nodes are distinct and so the existence of the last node proves that $\left(a_{1} \ldots a_{r}\right)^{m+1} a_{1} \ldots a_{p} \neq\left(a_{1} \ldots a_{r}\right)^{m+2} a_{1} \ldots a_{p}$. Then the identity $\alpha_{r}$ from (1) for $k$-testability is not valid on the transition semigroup of the automaton.

The lemma is proved.

Lemma 35 Suppose that on the 2-tuple graph of a deterministic finite locally testable automaton there exist an SCC-restricted path of length $k-1$. 
Then the identity $\beta$ (2) of $k$-testability is not valid on the transition semigroup $S$ of the automaton and both $S$ and the automaton are not $k$-testable.

$k+1$ is a lower bound on the order of local testability of the automaton

Proof. In order to prove the non-validity of the identity $\beta$ we must find elements $a_{1}, \ldots, a_{k-1}, b, c \in S$ such that

$$
a_{1} \ldots a_{k-1} b a_{1} \ldots a_{k-1} c a_{1} \ldots a_{k-1} \neq a_{1} \ldots a_{k-1} c a_{1} \ldots a_{k-1} b a_{1} \ldots a_{k-1}
$$

Let $(\mathbf{s}, \mathbf{q})$ be the first node on the considered path on $\Gamma \Gamma$ and elements $a_{1}, \ldots, a_{k-1} \in$ $S$ denote edges of the path. Let us denote $a=a_{1} \ldots a_{k-1}$. So the node $(\mathbf{s} a, \mathbf{q} a)$ is the last node on the path. Hence, $\mathbf{s} a \neq \mathbf{q} a$ and the nodes $\mathbf{s}, \mathbf{q}, \mathbf{s} a, \mathbf{q} a$ belong to one $S C C X$ of $\Gamma$. So there are elements $b, c \in S$ such that $\mathbf{s} a b=\mathbf{s}, \mathbf{s} a c=\mathbf{q}$. Without loss of generality let us assume that element $b$ is divided by an idempotent $e \in S$. This follows from the equality $s(a b)^{n}=s$ and local testability of $S$. Thus, $b=b_{1} e b_{2}$ for some $b_{1}, b_{2} \in S$.

If $\mathbf{s} a b_{1} e \not \mathbf{q} a b_{1} e$ from the fact that $\mathbf{s} a b a c a=\mathbf{s} a b_{1} e b_{2} a c a=\mathbf{q} a \in X$ it follows that $\mathbf{s} a b_{1} e \in X$ and $\mathbf{q} a b_{1} e \notin X$ because distinct $S C C$ are not connected with a loop.

Note that for any node $(\mathbf{p}, \mathbf{r})$ such that the nodes $\mathbf{p}, \mathbf{r}$ lie outside the $S C C$ $X$ and are reachable from $X$ and for any node $(\mathbf{s}, \mathbf{t})$ such that $\mathbf{s}, \mathbf{t} \in X$ we have $((\mathbf{p}, \mathbf{r}) \nsucc(\mathbf{s}, \mathbf{t}))$.

The node $\mathbf{q} a b_{1} e$ lies outside $X$, then $\mathbf{s} a c a b a=\mathbf{q} a b a$ does not belong to $X$ too. From $\mathbf{q} a \in X$ we have $\mathbf{q} a=\mathbf{s} a b a c a \neq \mathbf{s} a c a b a=\mathbf{q} a b a$, whence $a b a c a \neq a c a b a$.

So we may suppose that $\mathbf{s} a b_{1} e \sim \mathbf{q} a b_{1} e$ and $\mathbf{q} a b_{1} e \in X$. The nodes $\mathbf{q} a b_{1} e$ and $\mathbf{s} a b_{1} e$ have the common right unit $e$ and belong to the same $S C C$. From lemma 3.1 it follows that $\mathbf{s} a b_{1} e=\mathbf{q} a b_{1} e$. Then $\mathbf{s} a b=\mathbf{q} a b$ and $\mathbf{q} a b=\mathbf{s}$. This implies $\mathbf{s} a c a b a=\mathbf{q} a b a=\mathbf{s} a$. Now from $\mathbf{s} a b a c a=\mathbf{s} a c a=\mathbf{q} a$ and $\mathbf{q} a \neq \mathbf{s} a$ it follows that $\mathbf{s} a c a b a \neq \mathbf{s} a b a c a$ and $a b a c a \neq a c a b a$.

The lemma is proved.

Lemma 36 Suppose that on the 2-tuple graph $\Gamma \Gamma$ of a deterministic finite locally testable automaton with state transition graph $\Gamma$ there exist $S C C$-semirestricted path $\phi$.

Then the second components of all nodes of the path $\phi$ belong to one SCC of $\Gamma$ and no node of the path $\phi$ does not belong to some SCC of the 2-tuple graph $\Gamma \Gamma$.

Proof. For the first node $\left(\mathbf{p}_{1}, \mathbf{q}_{1}\right)$ and the last node $\left(\mathbf{p}_{i}, \mathbf{q}_{i}\right)$ of the path $\phi$ we have $\left(\mathbf{p}_{1}, \mathbf{q}_{1}\right) \succ\left(\mathbf{p}_{i}, \mathbf{q}_{i}\right)$ and $\mathbf{q}_{i} \succ \mathbf{q}_{1}$. Hence, $\mathbf{q}_{1} \sim \mathbf{q}_{i} \sim \mathbf{q}_{j}$ for any $j<i$.

Suppose that the considered path $\phi$ has a common node $(\mathbf{p}, \mathbf{q})$ with some $S C C$ of $\Gamma \Gamma$. Then for some element $e$ from transition semigroup $S$ we have $\mathbf{p} e=\mathbf{p}, \mathbf{q} e=\mathbf{q}, \mathbf{p} \succ \mathbf{q}$. Then the necessary condition of local testability (lemma 32) implies that for any $x \in S$ such that $\mathbf{q} x \succ \mathbf{q}$ we have $\mathbf{p} x \succ \mathbf{q} x$. Therefore the node $(\mathbf{p}, \mathbf{q})$ could not belong to an $S C C$-semirestricted path. 
Lemma 37 Suppose that on the 2-tuple graph $\Gamma \Gamma$ of a deterministic finite automaton with state transition graph $\Gamma$ there exist $S C C$-semirestricted path of length $k-1$.

Then the identity $\beta$ of $k$-testability is not valid on the transition semigroup $S$ of the automaton and both $S$ and the automaton are not $k$-testable.

$k+1$ is a lower bound on the order of local testability of the automaton.

Proof. In order to prove the non-validity of identity $\beta$ we must find elements $a_{1}, \ldots, a_{k-1}, b, c \in S$ such that for $a=a_{1} \ldots a_{k-1}$ we have abaca $\neq a c a b a$ (See $(3))$.

Let $a_{1}, \ldots, a_{k-1}$ denote the edges of the considered path $\left(\mathbf{p}_{1}, \mathbf{q}_{1}\right), \ldots,\left(\mathbf{p}_{k}, \mathbf{q}_{k}\right)$ and $a=a_{1} \ldots a_{k-1}$. Suppose that $\mathbf{p}_{k} \sigma \nsucc \mathbf{q}_{k} \sigma$ on $\Gamma$ for some $\sigma \in \Sigma$ such that $\mathbf{q}_{k} \sigma \succ \mathbf{q}_{1}$. From the preceding lemma and the definition of $S C C$-semirestricted path it follows that the nodes $\mathbf{q}_{1}, \mathbf{q}_{k}$ and $\mathbf{q}_{k} \sigma$ belong to one $S C C$ of $\Gamma$ and $\mathbf{p}_{k} \succ \mathbf{q}_{k}$, whence there exist an element $b \in S$ such that $\mathbf{p}_{k} b=\mathbf{q}_{1}$. By the abovementioned definition there exist an element $c=\sigma d \in S$ such that $\mathbf{q}_{k} c=\mathbf{q}_{1}$. Then $\mathbf{p}_{1} a b a c a=\mathbf{p}_{k} b a c a=\mathbf{q}_{1} a c a=\mathbf{q}_{k} c a=\mathbf{q}_{1} a=\mathbf{q}_{k}$. Consider the node $\mathbf{p}_{1} a c a b a=\mathbf{p}_{k} \sigma d a b a$. The node $\mathbf{q}_{k} \sigma$ is not reachable from $\mathbf{p}_{k} \sigma$ and so $\mathbf{p}_{k} \sigma \nsucc \mathbf{q}_{k}$, whence $\mathbf{p}_{k} \sigma d a b a \neq \mathbf{q}_{k}$. So $\mathbf{p}_{1} a b a c a \neq \mathbf{p}_{1} a c a b a$ and $a b a c a \neq a c a b a$.

The lemma is proved.

Lemma 38 Let $S$ be the transition semigroup of a locally testable reduced deterministic finite automaton and suppose that on the 2-tuple graph $\Gamma \Gamma$ of the automaton there are no strongly simple paths of length $k-1$. Suppose that $x \in S^{k-1}$, $y, z \in S$ and $S$ satisfies the identity $x y x=x y x y x$

Then $S$ satisfies identity $x y x z x=x z x y x$. (identity $\beta$ for $k$-testability)

Proof. From the identity $x y x=x y x y x$ we deduce the following identities

$$
x z x=x z x z x, x z x y x=x z x y x y x, x z x y x=x z x y x z x y x
$$

for $x \in S^{k-1}, y, z \in S$. So the words $x y x z x, x z x y x, x z x y x y, x y x z x z$ divide each other in $S$.

Let us suppose that the identity $x y x z x=x z x y x$ is not valid on $S$. Then for some node $\mathbf{p} \in \Gamma$ and for some $x \in S^{k-1}, y, z \in S$ we have $\mathbf{p} x z x y x \neq \mathbf{p} x y x z x$. Without loss of generality let us assume that there exists a node $\mathbf{p} x y x z x$.

Suppose first that $\mathbf{p} x \neq \mathbf{p} x y x z x$. Consider the path from the node $(\mathbf{p}, \mathbf{p} x y x z)$ to the node $(\mathbf{p} x, \mathbf{p} x y x z x)$ in $\Gamma \Gamma$. In view of $|x| \geq k-1$ some node on the path belongs to an $S C C$. The element $x$ may be presented in the form $x_{1} x_{2}$ such that the nodes $\mathbf{p} x_{1}$ and $\mathbf{p} x y x z x_{1}$ have a right unit in $\Gamma$. Now from the necessary condition of local testability (Lemma 32) it follows that $\mathbf{p} x s \succeq \mathbf{p} x y x z x s$ in $\Gamma$ for any $s \in S$ such that $x s$ is a left subword of the word of (4). Let $s=z x y x$. Then $\mathbf{p} x z x y x \succeq \mathbf{p} x y x z x z x y x=\mathbf{p} x y x z x y x$.

The equality $\mathbf{p} x y x z x=\mathbf{p} x y x z x y x z x$ follows from (4) and it implies that the nodes $\mathbf{p} x y x z x$ and $\mathbf{p} x y x z x y x$ belong to the same $S C C$ of $\Gamma$. Then in $\Gamma$ $\mathbf{p} x z x y x \succeq \mathbf{p} x y x z x$ and the first node of the formula exists. 
In the case that $\mathbf{p} x=\mathbf{p} x y x z x$ we have $\mathbf{p} x z x=\mathbf{p} x y x z x$ and $\mathbf{p} x z x=$ $\mathbf{p} x y x z x=\mathbf{p} x$. So $\mathbf{p} x=\mathbf{p} x z x y x z x$. Hence $\mathbf{p} x z x y x \succeq \mathbf{p} x y x z x$ and the node $\mathbf{p} x z x y x$ exist as well.

Now from the existence of the node $\mathbf{p} x z x y x$ it follows in analogous way that $\mathbf{p} x y x z x \succ \mathbf{p} x z x y x$. Thus, both nodes $\mathbf{p} x y x z x$ and $\mathbf{p} x z x y x$ belong to the same $S C C$.

The nodes pxyxzxz and pxzxyxy belong to the same $S C C$ as well. Multiplying by $x$ the nodes of one $S C C$ must unite them because the result belong to the same $S C C,|x| \geq k-1$ and on the path corresponding to $x$ there are no loops.

So $\mathbf{p} x y x z x z x=\mathbf{p} x z x y x y x$ for every $\mathbf{p} \in \Gamma$. Thus, $S$ satisfies the identity $x z x y x y=x y x z x z x$. In view of the identity $x y x=x y x y x$ we get that $x y x z x=$ $x z x y x$.

The lemma is proved.

Corollary. Let $S$ be the transition semigroup of a locally testable reduced deterministic finite automaton and suppose that on the 2-tuple graph $\Gamma \Gamma$ of the automaton there are no simple paths of length $k-1$. Suppose that $x \in S^{k-1}$, $y, z \in S$ and $S$ satisfies the identity $x y x=x y x y x$.

Then $S$ satisfies identity $x y x z x=x z x y x$. (identity $\beta$ for $k$-testability).

Lemma 39 Let $S$ be the transition semigroup of a locally testable reduced deterministic finite automaton and suppose that on the 2-tuple graph $\Gamma \Gamma$ of the automaton there are no SCC-restricted paths of length $k-1$. Suppose that $x \in S^{k-1}, y, z \in S$ and $S$ satisfies the identity $x y x=x y x y x$.

Then $S$ satisfies the identity $x y x z x=x y x z x y x$.

Proof. From the identity $x y x=x y x y x$ follow identities (4). This implies that the words $x y x z x, x y x z x y x, x y x z x y x y, x y x z x z$ are divided one by another. So the nodes pxyxzx, pxyxzxz, pxyxzxyx, pxyxzxyxy belong to a common $S C C$ of $\Gamma$. Suppose that $\mathbf{p} x y x z x z x \neq \mathbf{p} x y x z x y x y x$. Then on the 2tuple graph $\Gamma \Gamma$ there exists a path from the node (pxyxzxz, pxyxzxyxy) to the node $(\mathbf{p} x y x z x z x, \mathbf{p} x y x z x y x y x)$. We obtain a $S C C$-restricted path of the length $|x|=k-1$ This contradicts our assumption. So pxyxzxzx= pxyxzxyxyx. In view of (4) we have $\mathbf{p} x y x z x=\mathbf{p} x y x z x y x$.

The node $\mathbf{p}$ is an arbitrary node and so $x y x z x=x y x z x y x$.

The lemma is proved.

Theorem 310 Let $S$ be the transition semigroup of a reduced deterministic finite locally testable automaton $\mathbf{A}$ and $\Gamma \Gamma$ its 2-tuple graph. Assume the graph $\Gamma \Gamma$ does not contain simple paths of length $k-1$.

Then both the automaton $\mathbf{A}$ and the semigroup $S$ are $k$-testable.

$k$ is an upper bound on the order of local testability of the automaton 
Proof. The validity of the identities $\alpha_{r}$ for $k$-testability follows from lemma 33 . The validity of the identity $\beta$ in view of validity of $\alpha_{k}$ follows from corollary of lemma 38. .

From theorem 310 and lemmas 35, 37 we immediately obtain the following result.

Theorem 311 Let $\Gamma \Gamma$ be the 2-tuple graph of a locally testable deterministic reduced finite automaton $\mathbf{A}$. Let the maximum length of $S C C$-restricted and $S C C$-semirestricted paths on $\Gamma \Gamma$ be equal to $k-2$.

Then the identity $\beta$ of $(k-1)$-testability is not valid on the transition semigroup $S$ of the automaton $\mathbf{A}$ and both $S$ and the automaton are not $(k-1)$ testable, $k$ is a lower bound on the order of local testability.

If the length of all simple paths on $\Gamma \Gamma$ is not greater than $k-2$ then $\mathbf{A}$ is precisely $k$-testable.

Theorem 312 Assume that the state transition graph $\Gamma$ of a locally testable reduced deterministic finite automaton $\mathbf{A}$ is strongly connected. Let the maximum of the lengths of strongly simple [simple] paths on the 2-tuple graph of $\mathbf{A}$ be $k-2$.

Then the automaton is precisely $k$-testable.

The proof follows from the preceding theorem and from the fact that all paths on the 2-tuple graph of $\mathbf{A}$ are strongly simple, simple and $S C C$-restricted.

The determination of the order of local testability is in the general case NPhard [6]. But sometimes the situation is not so complicated.

Theorem 313 Let the state transition graph $\Gamma$ of a reduced deterministic finite automaton be strongly connected.

Then the order of local testability of the automaton may be found in polynomial time.

Proof. The verification of local testability is polynomial [4]. Finding the graph $\Gamma \Gamma$ and its diameter is polynomial too. According to the preceding theorem it gives us the answer.

\section{$4 \quad$ Necessary and sufficient conditions}

In this section we assume that for every node $\mathbf{q} \in \Gamma$ and every element $\sigma \in \Sigma$ the node $\mathbf{q} \sigma$ exist (the transition graph is complete). In general it is not very strong assumption because we can add to arbitrary graph $\Gamma$ a node $\mathbf{q}_{0}$ and suppose $\mathbf{q} \sigma=\mathbf{q}_{0}$ in all undefined cases. 
Lemma 41 Let $S$ be transition semigroup of a locally testable reduced deterministic finite automaton. Let on the 2-tuple graph $\Gamma \Gamma$ of the automaton there are no $S C C$-restricted and $S C C$-semirestricted paths of length $k-1$ and greater.

Let $x \in S^{k-1}, y, z \in S$ and $S$ satisfies identity $x y x=x y x y x$.

Then $S$ satisfies identity $x y x z x=x z x y x$ (Identity $\beta$ for $k$-testability).

Proof. Identity $x y x=x y x y x$ implies identities (4) and by lemma 39 it implies the identity $x z x y x=x z x y x z x$.

Let $\mathbf{p}$ be an arbitrary node of $\Gamma$.

Consider the nodes $\mathbf{p} x z x z$ and $\mathbf{p} x y x z x z$. In case $\mathbf{p} x z x z=\mathbf{p} x y x z x z$ we have $\mathbf{p} x z x y x=\mathbf{p} x z x z x y x==\mathbf{p} x y x z x z x y x$ and from lemma 39 and identity $x y x=$ $x y x y x$ it follows that $\mathbf{p} x z x y x=\mathbf{p} x y x z x$. This implies that $x z x y x=x y x z x$.

So let us suppose that $\mathbf{p} x z x z \neq \mathbf{p} x y x z x z$. Then the nodes $\mathbf{p} x$ and $\mathbf{p} x y x z x$ are distinct.

Let us suppose that $\mathbf{p} x z x z \nsucc \mathbf{p} x y x z x z$. Consider the path $\phi$ from the node $(\mathbf{p}, \mathbf{p} x y x z x z)$ to the node $(\mathbf{p} x, \mathbf{p} x y x z x z x)=(\mathbf{p} x, \mathbf{p} x y x z x)$ on $\Gamma \Gamma$. The length of the path is not less than $|x| \geq k-1$. Note that the nodes $\mathbf{p} x z x z, \mathbf{p} x y x z x z$ are reachable from the nodes $\mathbf{p} x$ and $\mathbf{p} x y x z x$ by help of the element $z x z$ and $\mathbf{p} x z x z \nsucc \mathbf{p} x y x z x z$. Therefore the path $\phi$ (or its part) is an $S C C$-semirestricted path of length $k-1$ or greater. This contradicts the condition of lemma.

So we may suppose that $\mathbf{p} x z x z \succ \mathbf{p} x y x z x z$ and $\mathbf{p} x y x y \succ \mathbf{p} x z x y x y$. Since the nodes $\mathbf{p} x z x z$ and $\mathbf{p} x y x z x z$ have the common unit $x z$, from necessary conditions of local testability (lemma 32) it follows that the node $\mathbf{p} x y x z x z x y x=\mathbf{p} x y x z x y x$ is reachable from the node $\mathbf{p} x z x z x y x=\mathbf{p} x z x y x$. In view of the lemma 39 we conclude that $\mathbf{p} x z x y x \succ \mathbf{p} x y x z x$. From $\mathbf{p} x y x y \succ \mathbf{p} x z x y x y$ it follows in analogous way that $\mathbf{p} x y x z x \succ \mathbf{p} x z x y x$.

So the nodes $\mathbf{p} x y x z x$ and $\mathbf{p} x z x y x$ belong to one $S C C$ of $\Gamma$. Then from (4) it follows that the nodes $\mathbf{p} x y x z x z$ and $\mathbf{p} x z x y x y$ belong to the same $S C C$. The length of $x$ is not less then $k-1$ and is greater then the length of every $S C C$ restricted path on $\Gamma \Gamma$. So $\mathbf{p} x y x z x z x=\mathbf{p} x z x y x y x$ and in view of $x y x=x y x y x$ we have $\mathbf{p} x y x z x=\mathbf{p} x z x y x$ in this case too.

Thus $x y x z x=x z x y x$.

The lemma is proved.

Lemma 42 Let $k$ be a maximal number such that on the 2-tuple graph $\Gamma \Gamma$ of deterministic finite locally testable reduced automaton $\mathbf{A}$ there exist $r$-periodic path of length $k+r$. Let $l$ be the maximum length of $S C C$-restricted paths on $\Gamma \Gamma$. Let $m$ be the maximum length of SCC-semirestricted paths on $\Gamma \Gamma$. Let $n>\max (k, l, m)+1$.

Then $\mathbf{A}$ is n-testable.

Proof. First consider the identities $\alpha_{r}$ of $n$-testability. Let us suppose that for some elements $a_{1}, \ldots, a_{r}$ from transition semigroup $S$ of the automaton

$$
\left(a_{1} \ldots a_{r}\right)^{m+1} a_{1} \ldots a_{p} \neq\left(a_{1} \ldots a_{r}\right)^{m+2} a_{1} \ldots a_{p}
$$

where $m r+p=n-1, p<r$. Then for some node $\mathbf{q} \in \Gamma$ we have $\mathbf{q}\left(a_{1} \ldots a_{r}\right)^{m+1} a_{1} \ldots a_{p} \neq$ $\mathbf{q}\left(a_{1} \ldots a_{r}\right)^{m+2} a_{1} \ldots a_{p}$. Hence, on the graph $\Gamma \Gamma$ there exist $r$-periodic path from 
the node $\left(\mathbf{q}, \mathbf{q} a_{1} \ldots a_{r}\right)$ of the length $(m+1) r+p=(m r+p)+r$. In view of equality $m r+p=n-1$ the length of the path is $n-1+r$. For $k=n-1$ we have $r$-periodical path of the length $k+r$. But it contradicts to our assumption that $n>\max (k, l, m)+1$ for all such $k$. So the identities $\alpha_{r}$ for $n$-testability hold in $S$.

The validity of identity $\beta$ follows from the preceding lemma.

The lemma is proved.

From the last lemma and lemmas 34, 35, 37 follow now the necessary and sufficient conditions for the order of local testability of deterministic finite reduced locally testable automaton.

Theorem 43 Let $k$ be the maximal natural number such that on the 2-tuple graph $\Gamma \Gamma$ of deterministic finite reduced locally testable automaton $\mathbf{A}$ there exist $r$-periodic path of length $k+r$. Let $l$ be the maximum length of all SCC-restricted paths on $\Gamma \Gamma$. Let $m$ be the maximum length of all SCC-semirestricted paths on $\Gamma \Gamma$. Let $n=\max (k, l, m)+2$.

Then $\mathbf{A}$ is precisely n-testable.

\section{The upper bound}

Lemma 51 Let $\Gamma \Gamma$ be the 2-tuple graph of locally testable deterministic finite automaton with $n$ states.

Then the length of any simple path on the graph $\Gamma \Gamma$ is at most $\frac{n^{2}-n}{2}-1$.

Proof. Any path on the graph $Г \Gamma$ could not contain both pairs $(\mathbf{p}, \mathbf{q})$ and $(\mathbf{q}, \mathbf{p})$ because it implies for some element $s$ of the transition semigroup that $\mathbf{q} s=\mathbf{p}$ and $\mathbf{p} s=\mathbf{q}$, whence some power of $s$ belongs to non-trivial group. But locally testable semigroup do not contain non-trivial subgroups [1].

The number of non-ordered pairs with distinct components on an $n$-element set is equal to $n(n-1) / 2$. Thus, the length of considered path is at most $n(n-$ 1) $/ 2-1$.

The lemma is proved.

Theorem 52 Let $S$ be the transition semigroup of a locally testable reduced deterministic finite automaton with $n$ states. Then both $S$ and the automaton are $\left(\frac{n^{2}-n}{2}+1\right)$-testable.

Proof immediately follows from theorem 310 and the preceding lemma.

\section{Example for the upper bound}

Let us consider the following example. Suppose the state transition graph $\Gamma$ of the finite automaton $\mathbf{M}$ contains $n$ nodes $\mathbf{q}_{1}, \ldots, \mathbf{q}_{n}$, for $n>2$. Let $\Sigma=\left\{a, b_{i, j}\right\}$, 
where $i=1, \ldots, n-2, n \geq j>i$. Suppose $\mathbf{q}_{3} a=\mathbf{q}_{1}$. For $k \neq 3 \mathbf{q}_{k} a$ is undefined. Suppose $\mathbf{q}_{i} b_{i, j}=\mathbf{q}_{i}, \mathbf{q}_{j} b_{i, j}=\mathbf{q}_{j+1}$ for all $i, j$ such that $i<j<n$ and for $i<n-1 \mathbf{q}_{i} b_{i, n}=\mathbf{q}_{i+1}, \mathbf{q}_{n} b_{i, n}=\mathbf{q}_{i+2}$. For other cases $\mathbf{q}_{k} b_{i, j}$ is undefined.

It will be proved that the automaton $\mathbf{M}$ is precisely $\left(\left(n^{2}-n\right) / 2+1\right)$-testable and so the upper bound of the order of testability from theorem 52 is obtainable.

Lemma 61 The state transition graph $\Gamma$ of the finite automaton $\mathbf{M}$ is strongly connected. $\mathbf{M}$ is locally testable.

Proof. In view of $\mathbf{q}_{1}=\mathbf{q}_{3} a, \mathbf{q}_{i} b_{j, i}=\mathbf{q}_{i+1}$ and $\mathbf{q}_{n} b_{1, n}=\mathbf{q}_{3}$ the graph $\Gamma$ is strongly connected and all nodes of $\Gamma$ belong to one $S C C$.

In [5] are given two conditions of local testability. First is the validity of the lemma 3.1 on $\Gamma$. Second must be verified only in case $\Gamma$ is not an $S C C$. Thus according to lemma 3.1 we must prove only that the distinct nodes of $\Gamma$ have no common unit in the transition semigroup $S$ of $\mathbf{M}$.

Suppose $\mathbf{p} x=\mathbf{p}, \mathbf{q} x=\mathbf{q}, \mathbf{p} \neq \mathbf{q}$ for $\mathbf{p}, \mathbf{q} \in \boldsymbol{\Gamma}, x \in S$. Since there exists only one element of the kind $\mathbf{q}_{i} a$ the element $x$ is not divided by $a$. So $x$ is a product of the $b_{i, j}$.

From $\mathbf{p} x=\mathbf{p} \neq \mathbf{q} \mathbf{x}=\mathbf{q}$ it follows that there is a cycle on the 2-tuple graph $\Gamma \Gamma$ and all edges of the cycle are denoted by $b_{i, j}$. Consider some node $\left(\mathbf{q}_{i}, \mathbf{q}_{j}\right)$ on the cycle. Suppose first $i>j$. Consider any existing node $\left(\mathbf{q}_{i}, \mathbf{q}_{j}\right) b_{l, r}=\left(\mathbf{q}_{i i}, \mathbf{q}_{j j}\right)$. So $r=i, l=j$. We have either $\left(\mathbf{q}_{i}, \mathbf{q}_{j}\right) b_{r, l}=\left(\mathbf{q}_{i+1}, \mathbf{q}_{j}\right)$ or in the case $i=n$ we have $\left(\mathbf{q}_{i}, \mathbf{q}_{j}\right) b_{l, r}=\left(\mathbf{q}_{j+2}, \mathbf{q}_{j+1}\right)$. Thus from $i>j$ it follows that $i i>j j, j j \geq j$ and in the case $j j=j$ we have $i i>i$. So $j j * n+i i>j * n+i$.

Multiplication on $b_{l, r}$ induces a lexicographical order on the pairs $(\mathbf{p}, \mathbf{q})$ and all nodes on the path with edges $b_{l, r}$ are distinct. So our assumption in the case $i>j$ is not true.

In the case $i<j$ we obtain contradiction too.

Thus $\mathbf{p} x=\mathbf{p}, \mathbf{q} x=\mathbf{q}$ implies $\mathbf{p}=\mathbf{q}$. Therefore $\mathbf{M}$ is locally testable.

Lemma 62 On the 2-tuple graph $\Gamma \Gamma$ of the automaton $\mathbf{M}$ there exists an $S C C$-restricted path of length $\frac{n^{2}-n}{2}-1$.

Proof. Consider the path: $\left(\mathbf{q}_{1}, \mathbf{q}_{2},\right),\left(\mathbf{q}_{1}, \mathbf{q}_{3}\right), \ldots,\left(\mathbf{q}_{1}, \mathbf{q}_{n}\right),\left(\mathbf{q}_{2}, \mathbf{q}_{3}\right), \ldots\left(\mathbf{q}_{2}, \mathbf{q}_{n}\right)$, $\ldots\left(\mathbf{q}_{n-2}, \mathbf{q}_{\mathbf{n}}\right),\left(\mathbf{q}_{\mathbf{n}-\mathbf{1}}, \mathbf{q}_{\mathbf{n}}\right)$. The nodes of the path are connected with edges noted by $b_{i, j}$. All nodes of the kind $\left(\mathbf{q}_{i}, \mathbf{q}_{j}\right)$ such that $i<j$ belong to the path one time. The number of such nodes is $\frac{n^{2}-n}{2}$, so the length of the path is $\frac{n^{2}-n}{2}-1$. In view of the preceding lemma it is $S C C$-restricted path.

Theorem 63 Deterministic finite automaton $\mathbf{M}$ with $n$ states $(n>2)$ is precisely $\left(\frac{n^{2}-n}{2}+1\right)$-testable and its order of local testability is equal to the upper bound on the order of local testability of a deterministic finite reduced automaton with $n$ states.

Proof. Lemma 61 gives us the local testability of M. From theorem 52 follows that for $\mathbf{M}$ the upper bound of order of local testability is equal to $\left(n^{2}-n\right) / 2+1$. Lemma 35 in view of lemma 62 implies that the upper bound is reached on $\mathbf{M}$. 
The theorem is proved.

This implies the validity of the following statement

Theorem 64 The precise upper bound on the order of local testability of deterministic finite locally testable reduced automata with $n$ states is equal to $\frac{n^{2}-n}{2}+1$

Proof. For $n>2$ it follows from the preceding theorem. For $n=2$ the semigroup of left zeroes gives us the needed example.

\section{Example for two variables}

Let us consider the following example of the state transition graph $\Gamma$ of the finite deterministic automaton $\mathbf{M}$ :

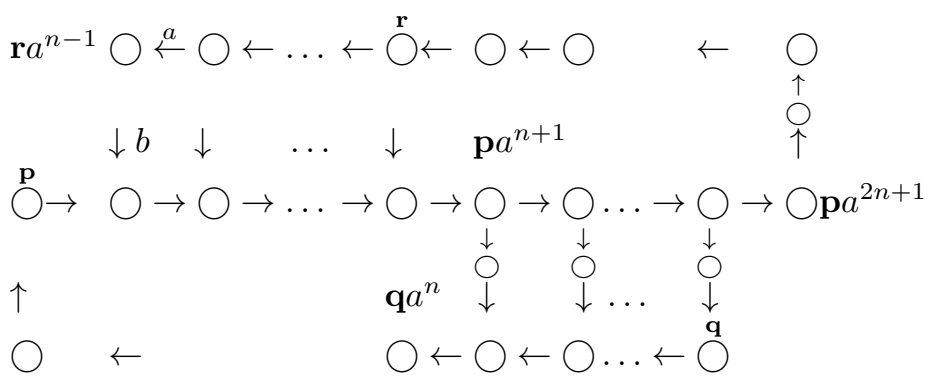

The vertical edges are noted by $b$, the horizontal edges are noted by $a$.

We have for $i<n$

$$
\begin{gathered}
\mathbf{p} a^{2 n} b^{2}=\mathbf{q}, \mathbf{p} a^{n+1} b^{2}=\mathbf{q} a^{n-1}, \mathbf{q} a^{n+1} b=\mathbf{p}, \mathbf{p} a^{n+i} b^{2}=\mathbf{q} a^{n-i} \\
\mathbf{r} b=\mathbf{p} a^{n}, \mathbf{r} a^{n-i} b=\mathbf{p} a^{i}, \mathbf{r} a^{n-1} b=\mathbf{p} a, \mathbf{p} a^{2 n+1} b^{2} a^{3}=\mathbf{r}
\end{gathered}
$$

So

$\mathbf{p} a=\mathbf{p} a^{2 n+1} b^{2} a^{n+2} b, \mathbf{p}=\mathbf{p} a^{2 n} b^{2} a^{n+1} b, \mathbf{p}=\mathbf{p} a^{2 n-i} b^{2} a^{n+1-i} b, \mathbf{p} a^{i}=\mathbf{p} a^{2 n+1} b^{2} a^{n+3-i} b$.

On the middle line there are $2 n+2$ nodes, on the top line there are $n+3$ nodes, on the bottom line there are $n+2$ nodes.

It will be proved that the order of local testability of the automaton $\mathbf{M}$ is $\Omega\left(n^{2}\right)$.

Obvious is the following

Lemma $\mathbf{7 1}$ The state transition graph $\Gamma$ of the finite deterministic automaton $\mathbf{M}$ is strongly connected ( $\mathbf{M}$ is an $S C C$ ).

Lemma $\mathbf{7 2}$ The finite deterministic automaton $\mathbf{M}$ is locally testable. 
Proof. In [5] are given two conditions for the automaton to be locally testable. First is the validity of lemma 3.1 on $\Gamma$. Second must be verified only in the case that $\Gamma$ is not an $S C C$. Thus, by the preceding lemma, we must prove only that distinct nodes of $\Gamma$ have no common unit in transition semigroup $S$ of $\mathbf{M}$.

Suppose that there are two cycles on $\Gamma$ with edges corresponding the element $x=a^{k_{1}} b^{l_{1}} a^{k_{2}} b^{l_{2}} \ldots a^{k_{s}} b^{l_{s}}$ from transition semigroup $S$ of $\mathbf{M}$. Our aim is to prove that both the cycles coincide.

Let us assume that for nodes $\mathbf{f}, \mathbf{g} \in \Gamma$ we have $\mathbf{f} x=\mathbf{f}, \mathbf{g} x=\mathbf{g}, \mathbf{f} \neq \mathbf{g}$.

Let $x_{i}$ be the left subword of the word $x$ of length $i$.

Then $\mathbf{f} x_{i} \neq \mathbf{g} x_{i}$ for any $i$ and there exist a cycle in the 2-tuple graph $\Gamma \Gamma$ with the nodes $\left(\mathbf{f} x_{i}, \mathbf{q} x_{i}\right), 0<i \leq|x|$.

It is not difficult to see that $l_{j}=1$ or $l_{j}=2$ and $l_{j}+l_{j+1}=3$. Without loss of generality we can assume that $l_{1}=2$. The nodes $\mathbf{f} a^{k_{1}} b^{2}=\mathbf{f} x_{k_{1}+2}$ and $\mathbf{g} a^{k_{1}} b^{2}=\mathbf{g} x_{k_{1}+2}$ exist only if $\mathbf{f} x_{k_{1}}=\mathbf{p} a^{m}$ and $\mathbf{g} x_{k_{1}}=\mathbf{p} a^{l}$, for some $m, l>n$. Both the nodes $\mathbf{f} a^{k_{1}} b^{2} a^{k_{2}} b$ and $\mathbf{g} a^{k_{1}} b^{2} a^{k_{2}} b$ exist and are distinct. They belong to the middle line and are presented in the form $\mathbf{p} a^{i}(i \geq 0)$. Since not more than one of them may be $\mathbf{p}$, another is equal to $\mathbf{p} a^{i}$ where $i \geq 1$. Let us suppose that $\mathbf{f} a^{k_{1}} b^{2} a^{k_{2}} b=\mathbf{p} a^{i}$ for $i>0$. Then $\mathbf{f} a^{k_{1}} b^{2}=\mathbf{p} a^{2 n+1}$ and $\mathbf{g} a^{k_{1}} b^{2}=\mathbf{p} a^{n+t}$ where $0<t<n+1$. So to the cycle of $\Gamma \Gamma$ belongs the node $\left(\mathbf{p} a^{2 n+1}, \mathbf{p} a^{n+t}\right)$. Then the node $\left(\mathbf{p} a^{2 n+1} b^{2}, \mathbf{p} a^{n+t} b^{2}\right)$ belongs to the same cycle. It implies that the node $\left(\mathbf{p} a^{2 n+1} b^{2} a^{3}, \mathbf{p} a^{n+t} b^{2} a^{3}\right)=\left(\mathbf{r}, \mathbf{q} a^{n-t+3}\right)$ is on the same cycle too. The second component of one of the nodes on considered cycle of $\Gamma \Gamma$ must to be $\mathbf{p}$. From $\mathbf{q} a^{n-t+3} a^{j} b=\mathbf{p}$ follows that $j+n-t+3=n+1$ and $j=t-2$. Then the first component of the same node is $\mathbf{r} a^{j} b=\mathbf{p} a^{n-j}$. From the node $\left(\mathbf{p} a^{2 n+1}, \mathbf{p} a^{n+t}\right)$ we reach the node $\left(\mathbf{p} a^{n-t+2}, \mathbf{p}\right)$ and therefore the node $\left(\mathbf{p} a^{2 n+1}, \mathbf{p} a^{n+t-1}\right)$.

Distance between components of the nodes is growing from $a^{n-t+1}$ to $a^{n-t+2}$. So for subword of $x$ containing two distinct inclusions of $b\left(b^{2}\right.$ and then $\left.b\right)$ distance between components is growing. Obvious that $s$ is even number. So the distance between two components of the node is growing on the path corresponding $x$. This contradicts to the fact that $x$ defines the cycle on $\Gamma \Gamma$ (Or two distinct corresponding cycles on $\Gamma$ ).

So $\mathbf{M}$ is locally testable.

Lemma 73 On the 2-tuple graph $Г \Gamma$ of the automaton $\mathbf{M}$ there exist a $S C C$ restricted path of length $2 n^{2}+4 n-6$.

Proof. Consider the path defined by the word $a^{2 n+1-i} b^{2} a^{n+2-i} b$ from the node $\left(\mathbf{p}, \mathbf{p} a^{i}\right)$ for $0<i<n$. We have

$$
\left(\mathbf{p}, \mathbf{p} a^{i}\right) a^{2 n+1-i} b^{2} a^{n+2-i} b=\left(\mathbf{p}, \mathbf{p} a^{i+1}\right) .
$$

The length of the path is equal to $3 n+6-2 i$, the final node is $\left(\mathbf{p}, \mathbf{p} a^{i+1}\right)$.

Now consider the sequence of such paths for $i=1,2, \ldots, n-1$. We get a path from the node $(\mathbf{p}, \mathbf{p} a)$ to the node $\left(\mathbf{p}, \mathbf{p} a^{n}\right)$. The length of the path is $2 n^{2}+4 n-6$.

The lemma is proved.

Theorem $\mathbf{7 4}$ Deterministic finite automaton $\mathbf{M}$ whose alphabet size is two is locally testable and its order of local testability is $\Omega\left(n^{2}\right)$. 
Proof. Lemma 72 gives us the local testability of $\mathbf{M}$. Number of nodes in $\mathbf{M}$ is equal to $5 n+8$ and is linear in $n$. According the preceding lemma there exist a path of length $2 n^{2}+4 n-6$ on the 2-tuple graph of the automaton. In view of lemma 35, this number gives us a lower bound for the order of local testability.

So the lower bound for the order of local testability is $\Omega\left(n^{2}\right)$. According theorem 52 (see [5] too) it is an upper bound as well.

\section{Acknowledgments}

The author thanks professor Margolis for helpful discussion and for many useful suggestions.

\section{References}

1. J.A. Brzozowsky, I. Simon, Characterization of locally testable events, Discrete Math., 4, (1973) 243-271.

2. A. Ginzburg, About some properties of definite, reverse-definite and related automata, IEEE Trans. Electron. Comput. ES15(1966) 806-810.

3. P. Caron, AG: Families of locally testable languages. Rapport LIR 97.03.Univ. de Rouen,Fr. 1997, 206-210.

4. S. Kim, R. McNaughton, R. McCloskey, An upper bound on the order of locally testable deterministic finite automaton, Lect. Notes in Comp., 401, (1989) 48-65.

5. S. Kim, R. McNaughton, R. McCloskey, A polynomial time algorithm for the local testability problem of deterministic finite automata, IEEE Trans. Comput. 40(1991) N10, 1087-1093.

6. S. Kim, R. McNaughton, Computing the order of locally testable automaton, SIAM J. Comput., 23(1994), 1193-1215.

7. G. Lallement, Semigroups and combinatorial applications, Wiley, N.Y., 1979

8. S. W. Margolis, J.E.Pin. Languages and inverse semigroups, 11 ICALP, Lect. Notes in Comp. Sc, 199, Springer, Berlin(1985) 285-299.

9. R. McNaughton, S. Papert, Counter-free automata, M.I.T. Press Mass., 1971.

10. M. Minsky, S. Papert, Perceptrons, M.I.T. Press Mass., 1971, Cambridge, MA, 1969.

11. Pastijn, F., Regular locally testable semigroups as semigroups of quasi-ideals, Acta Math. Hung., 36, 1-2(1980), pp. 161-166.

12. Pin J., Finite semigroups and recognizable languages. An introduction. Semigroups and formal languages, Math. and Ph. Sc., v.466, (1995), pp. 1-32.

13. M. Perles, M. O. Rabin, E. Shamir, The theory of definite automata, IEEE Trans. Electron. Comput. ES-12(1963) 233-243.

14. A.N. Trahtman, The varieties of testable semigroups. Semigroup Forum, 27, (1983), 309-318.

15. A.N. Trahtman, Precise upper bound on the order of local testability of finite automaton. 2 Int. Workshop on Impl. Automata, Univ. of Western Ontario, Canada, 1997, 113-121.

16. Y. Zalcstein, Locally testable semigroups, Semigroup Forum, 5, (1973), 216-227.

17. Zalcstein, Y., Syntactic semigroups of some classes of star-free languages, J. Comp. System Sci., 6, (1972), pp. 151-167. 